\title{
Standardized diagram for rapid tabulation of coronary arteriograms
}

\author{
Marshall Franklin, Michael V. O'Reilly, ${ }^{1}$ and Martin J. Krauthamer \\ From Norwalk Hospital, Norwalk, Connecticut, U.S.A.
}

A concise, easy to read system of recording the results of coronary arteriography using a diagram and symbols is presented with a number of descriptive examples. This system has proved itself invaluable in a busy laboratory (40-50 cases a month) over the past 2 years.

The precise and detailed recording of the results of coronary arteriography requires a lengthy and involved description of lesions, and, where present, collateral channels. To this must be added the status of the myocardium in the distribution of the diseased vessels, details of the adequacy of collateral flow, and the presence or absence of lesions in the parent vessels providing the collaterals.

To view all these details clearly and in true perspective when recorded in words is impossible: one is usually forced to review the angiograms which may not be immediately available. To overcome the problem and facilitate an easy, complete, and rapid recording of the overall picture on a filing card for each patient, we have (in the course of over 1500 arteriograms) evolved the following system which seems far superior to our previous method of tabulating coronary arteriograms, and accordingly present it here for the benefit of others.

\section{Principle}

The information is stored on $5 \times 8$ file cards. The left side is occupied by a large skeleton diagram of the coronary arterial system, the right by a line drawing of the left ventricle in the right anterior oblique projection, the space below by a tabulation of the lesions, with collaterals where present, and a notation concerning myocardial integrity in the distribution of the vessel.

The relative positions of all the vessels on the coronary artery skeleton are represented as they would appear on the angiograms at $45^{\circ}-60^{\circ}$ rotation in the left anterior oblique projection except for the distal circumflex and its branches, which are shown in outline as the mirror image of their appearance in $25^{\circ}-30^{\circ}$ right anterior oblique projection. In addition, the anterior descending

Received 26 June 1972.

1 Present address: Department of Physiology, Royal College of Surgeons in Ireland, St. Stephen's Green, Dublin 2, Eire. system is spread out rather like it would appear with the patient rotated to about $75^{\circ}$ left anterior oblique.

These modifications have the advantage of both spreading out the right and circumflex systems such that their posterior atrioventricular branches can be represented as one gentle sweeping arc, with the usual posterior descending, posterior left ventricular, and posterolateral left ventricular offshoots. At the same time, branches which are commonly interconnected by collateral channels come to lie adjacent to one another. The sinuatrial and atrioventricular nodes are inserted at the upper and lower borders respectively near the midportion of the chart. The point of division between the right and circumflex systems is easily inserted at the relevant location depending on the degree of balance/dominance in each individual case.

\section{Symbols and labelling}

Patent vessels are inked in over the faint line diagram using a felt pen. The four main trunks (left main stem, anterior descending, circumflex, and right coronary artery) are labelled at their origin, and subsequent branches are labelled at their termination. Branches normally variable in size are labelled using capitals or lower case letters depending on their magnitude. The heavy line is discontinued at the site of a lesion where numbers $(I-4,9, x)$ are inserted in ball pen representing the severity of the lesion (Table I). A discrete or lengthy lesion is differentiated by a square or circle around this number, the number alone representing generalized narrowing throughout the entire course of the vessel. Collaterals are drawn (in red) by ball pen using a saw-toothed line continuous if adequate with good flow, or interrupted if faint and inadequate. Vessels which opacify only very faintly are represented by a dotted line in felt pen.

In Table 2 listing the lesions, collaterals, and myocardial integrity, the same symbols and abbreviations are used as appear on the diagram. The extreme left column on the file card lists lesions without a collateral supply. The second column is for vessels which receive collateral flow, the parent vessel providing the collateral 
TABLE I Abbreviations for coronary arteries and their branches

\begin{tabular}{ll}
\hline Symbol & Major vessels \\
R & Right coronary artery \\
L & Left coronary artery main trunk \\
C & Circumflex branch \\
A & Anterior descending branch \\
& Branches of right coronary system \\
AM & Acute marginal branch of right \\
am & Anterior marginal branch of right \\
Co & Conus branch of right \\
& Branches of left coronary system \\
D & Diagonal branch of left \\
IV & Interventricular segment of left \\
SP & Large septal perforator of left \\
sp & Small septal perforator of left \\
LM & Lateral marginal branch of circumflex \\
AlLV & Anterolateral left ventricular branch of \\
plLV & circumflex \\
& Posterolateral left ventricular branch of \\
& circumflex \\
PD & Variable branches \\
PLV & Posterior descending branch \\
PAV & Posterior left ventricular branch \\
pav & Large posterior atrioventricular branch \\
pat & Small posterior atrioventricular branch \\
\hline & Posterior atrial branch \\
\hline
\end{tabular}

\section{TABLE 2 Abbreviations}

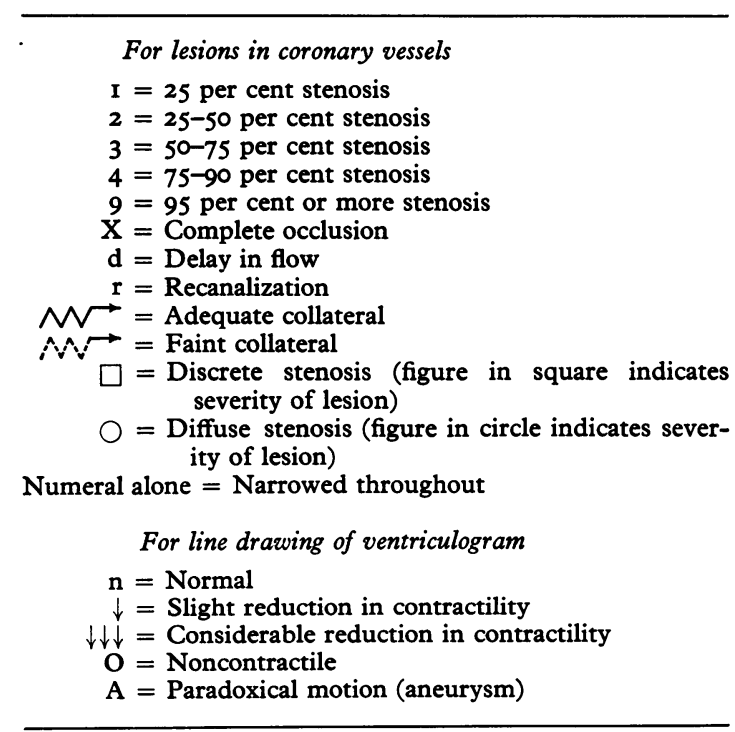

appearing on the same line either to the left in the 'non collat' column if severely constricted, or in the third column on the right if the parent vessel is healthy. The fourth column notes the integrity of the ventricular

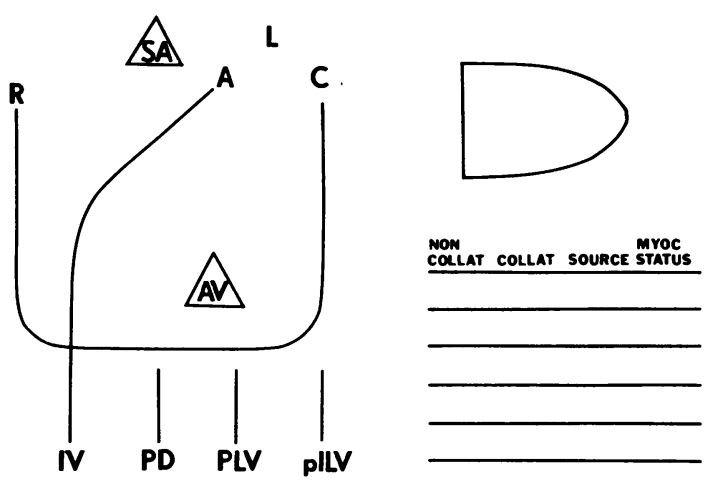

FIG. I The skeleton diagram: for details see description in text.

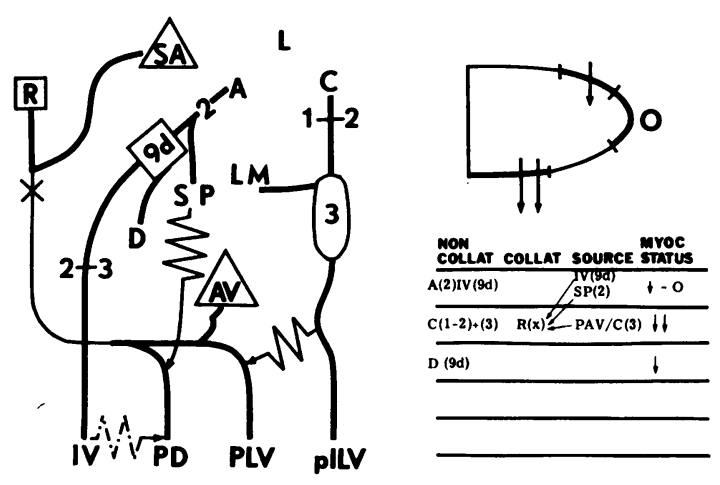

FIG. 2 The completed diagram: for details see description in text.

myocardium in the distribution of the vessels involved. Clinical information, symptoms, physical findings, etc., are listed on the reverse side. Two specimen cards are presented (Fig. I and 2).

\section{Description of diagrams}

Fig. I shows the skeleton diagram. The central feature is the U-shaped outline of the main trunks of the right and circumflex vessels connected by their atrioventricular branches. Inside of this is situated the (at first oblique and then vertical) outline of the anterior descending artery and its interventricular continuation. Lying below and to the right of the distal part of the interventricular segment are the posterior descending, posterior left ventricular, and posterolateral left ventricular branches of the atrioventricular groove segment. The atrioventricular and sinuatrial nodes are shown in the midline at the lower and upper areas, respectively.

To the right is situated the line diagram of the left ventricle in the right anterior oblique projection and the headings for the tabulated summary of the coronary lesions comprising columns for noncollateralized vessels, 
collateralized vessels, source of the collaterals, and the myocardial status in the distribution of the lesions.

Fig. 2 shows the completed diagram. From left to right: The right coronary artery is dominant as noted by the square around the letter at its origin. This vessel is completely obstructed in its proximal third after the origin of the artery to the sinuatrial node, as denoted by the $\mathrm{X}$. The patent segment is detectable by the thick line added to the skeleton with a felt pen. The middle section of this vessel does not opacify but the posterior descending and posterior left ventricular branches do, as denoted by increased thickness of the outline superimposed on the skeleton. These vessels are filled by collateral channels from the interventricular and septal perforating branches, of the anterior descending, and the posterolateral left ventricular branch, which arises from the circumflex in this case, respectively. The saw tooth lines added to the skeleton diagram record the collaterals. All the remaining vessels are patent, and this is noted by the heavier outline of their course. The diagonal and septal perforating branches of the anterior descending and the lateral marginal branch of the circumflex have been added to the skeleton diagram. The following lesions have been noted. The diffuse irregularity and constriction of 25 to 50 per cent of the main trunk of the anterior descending as noted by the figure 2 . A discrete 95 per cent stenosis of the anterior descending at the origin of the diagonal branch with subsequent delay in filling of these vessels as denoted by the $9 \mathrm{~d}$ in a square. The remainder of the intraventricular segment appears irregular and narrowed by 50 to 75 per cent as shown by the figures 2-3 in the middle of its course. The collaterals from the IV segment are faint-inadequate, hence the broken line.

Similarly the proximal portion of the circumflex is irregular and narrowed by 25 to 50 per cent - I-2 superinscribed near its origin; while encompassing and distal to the origin of the left marginal branch there is a rather diffuse stenosis of 50 to 75 per cent represented by the figure 3 in an oval.

Lastly, the main trunk of the left is free of disease and the artery to the atrioventricular node arises from the dominant right system. Turning our attention to the line diagram of the left ventricle there is noted a moderately dyskinetic area in the inferobasal region, a mildly dyskinetic area in the distal portion of the anterior wall, and the apex is noncontractile.

This information is summarized in symbols on the columns below the left ventricular outline. The noncol- lateralized lesions, that is the severe stenosis of the anterior descending and intraventricular segments at the origin of the diagonal denoted in column $I$, the collateralized distal portion of the obstructed right is tabulated in the second column and the source of these collaterals which are healthy noted in the third column (from the posterior atrioventricular groove branch of the circumflex and the septal perforating branch of the anterior descending). Lastly, the fourth column shows the status of the myocardium in the distribution of these lesions, being noncontractile in the areas supplied by the anterior descending, moderately dyskinetic in the distribution of the obstructed right, and mildly dyskinetic in the distribution of the diagonal branch.

Once familiarity with this system is obtained all this information can be obtained at a glance at the above file cards, and a rapid, accurate, and complete report provided to an inquirer with minimum delay.

\section{Discussion}

Although this system may seem involved initially, we have found it to be of great advantage once familiar with it. No doubt further modification will evolve as experience increases to meet changing circumstances. This technique has proved to be extremely useful, providing at a glance the total picture of the patient's overall status. The information is obtained from a single $5 \times 8$ card without need to refer to the original films. A further unexpected benefit of the introduction of this system has been a higher standard of film interpretation resulting from the increased attention to detail required to incorporate all information on the diagram. There also has been less interobserver variation and a reduced incidence of overlooked minor lesions. In addition, the dictation of reports mainly from the coronary diagram without constant referral to the angiogram has resulted in easier transcription from the dictaphone and improved continuity and clarity of the written reports.

Requests for reprints to Dr. M. O'Reilly, Department of Physiology, Royal College of Surgeons in Ireland, St. Stephen's Green, Dublin 2, Eire. 